Jussara Smidt Porto, Clarissa Coussirat Angrizani, Carmen

Iara Walter Calcagno, Lauren da Cunha Duarte *

\title{
Estudo sobre a impermeabilização do compósito amido / resíduo de papel
}

Jussara Smidt Porto é Designer Gráfica. Ela é servidora pública lotada na Gráfica UFRGS. Durante sua trajetória na universidade, como diretora da Gráfica da UFRGS, adquiriu experiência na produção gráfica, gestão de pessoas, ecodesign, coordenação de projetos de extensão, visando melhorias dos processos produtivos da Gráfica, sustentabilidade e inovação. E esse conhecimento adquirido foi fundamental para que ela desenvolvesse o seu doutorado na própria Universidade Federal do Rio Grande do Sul (UFRGS) no programa de Pós-Graduação em Design, na qual pesquisou possíveis soluções para o reaproveitamento do resíduo de papel descartado pela Gráfica UFRGS. <00006708@ufrgs.br> ORCID: 0000-0002-3843-4934
Resumo Esta pesquisa apresenta um material alternativo que segue padrões inovadores e sustentáveis constituído pela união da matriz de amido gelatinizado com carga de resíduo de papel. Considerando que o amido e o papel possuem natureza hidrofílica, podem ocorrer limitações nas aplicações deste biocompósito caso não seja criada uma barreira para restringir a absorção de umidade. Para tanto, foram realizados testes de impermeabilização a fim de analisar o comportamento do material em relação à absorção de água (2 e 24 horas) com quatro diferentes impermeabilizantes com uma, duas e três demãos. Foram obtidos resultados satisfatórios a partir da segunda demão em $2 \mathrm{~h}$ e terceira demão em $24 \mathrm{~h}$, com os impermeabilizantes à base de poliuretano. Portanto, comparando-se aos outros impermeabilizantes testados, e buscando-se resultados mais sustentáveis, pode-se concluir que o impermeabilizante à base de poliuretano de origem vegetal demonstrou ser o que melhor atende aos objetivos desta pesquisa.

Palavras chave Biocompósito, Amido, Resíduo de papel, Impermeabilizantes, Absorção de água. 
Clarissa Coussirat Angrizani é engenheira mecânica pelo Instituto Federal de Educação, Ciência e Tecnologia Sul-Rio-Grandense (IFSUL) no campus Sapucaia do Sul, e possui doutorado em engenharia de materiais pela Universidade Federal do Rio Grande do Sul (UFRGS). Atualmente desenvolve pesquisas no desenvolvimento e caracterização de compósitos poliméricos reforçados com fibras vegetais e resíduos industriais. <cangrizani@gmail.com> ORCID: 0000-0002-6216-9712

\section{Study about the waterproofing of the composite starch / paper waste}

Abstract This research presents an alternative material that follows innovative and sustainable standards constituted by the union of the gelatinized starch matrix with paper waste as load. Since starch and paper are hydrophilic nature, limitations in the applications of this biocomposite may occur if no barrier is created to restrict moisture absorption. For this, waterproofing tests were performed to analyze the behavior of the material in relation to water absorption (2 and 24 hours) with four different waterproofing agents applying one, two and three layer. Satisfactory results were obtained from the second coat in $2 \mathrm{~h}$ and third coat in $24 \mathrm{~h}$ with the polyurethane based waterproofing agents. Therefore, comparing to the other waterproofing agents tested, and searching for more sustainable results, it can be concluded that the polyurethane-based waterproofing agent of vegetable origin proved to be the one that best meets the objectives of this research.

Keywords Biocomposite, Starch, Paper waste, Waterproofing, Water absorption.

Carmen Iara Walter Calcagno é quí dos materiais. Desde 1998, atua como professora do Instituto Federal Sul-Rio-Grandense (IFSUL) no campus Sapucaia do Sul. Realiza pesquisa em desenvolvimento de produtos e processos na área de materiais poliméricos e cerâmicos Em 2018 publicou o livro "Plásticos" que aborda conhecimento sobre polímeros e processamento de termoplásticos. <carmencalcagno@sapucaia.ifsul.edu.br > ORCID: 0000-0001-8407-6675

Lauren da Cunha Duarte é geóloga e doutora em geociências. Desde 2009 é professora Associada da Universidade Federal do Rio Grande do Sul (UFRGS), pelo Departamento de Mineralogia e Petrologia, sendo docente permanente junto ao programa de Pós-Graduação em Design e Tecnologia/UFRGS atuando na área de pesquisa de Materiais Naturais nos temas de beneficiamento, melhoramento e caracterização de materiais naturais.<lauren.duarte@ufrgs.br> ORCID: 0000-0001-5690-0794

\section{Estudio sobre la impermeabilización del compuesto de almidón / papel de desecho}

Resumen Esta investigación presenta un material alternativo que sigue estándares innovadores y sostenibles constituidos por la unión de la matriz de almidón gelatinizado con una carga de residuos de papel. Teniendo en cuenta que el almidón y el papel tienen una naturaleza hidrófila, puede haber limitaciones en las aplicaciones de este biocompuesto si no se crea una barrera para restringir la absorción de humedad. Para este fin, se realizaron pruebas de impermeabilización para analizar el comportamiento del material en relación con la absorción de agua ( 2 y 24 horas) con cuatro agentes impermeabilizantes diferentes con una, dos y tres capas. Se obtuvieron resultados satisfactorios de la segunda capa en 2 horas y la tercera capa en 24 horas, con productos impermeabilizantes a base de poliuretano. Por lo tanto, en comparación con la otra impermeabilización probada y buscando resultados más sostenibles, se puede concluir que la impermeabilización a base de poliuretano a base de plantas demostró ser la que mejor cumple con los objetivos de esta investigación.

Palabras clave Biocompuesto, Almidón, Residuos de papel, Impermeabilización, Absorción de agua. 


\section{Introdução}

Devido a problemas ambientais e as novas exigências de mercado, cada vez mais tem se intensificado o desenvolvimento de materiais biocompósitos (FARUK et al., 2012), constituindo-se como um avanço importante para a história da engenharia dos materiais (SANJAY et al., 2015). Para a elaboração destes biocompósitos, utilizam-se os mais diversos materiais, tais como, as fibras vegetais e alguns resíduos industriais que são empregados como reforço em materiais poliméricos.

Os resíduos sólidos industriais são uma grande preocupação ambiental que demandam atenção. A possibilidade da aplicação dos 3Rs (reduzir, reutilizar e reciclar), para a sua redução pode ser um dos caminhos para a melhoria das condições ambientais e promover uma relação mais harmônica entre o ambiente natural e o consumidor (PICCOLI et al., 2014).

Seguindo esta tendência ecológica, esta pesquisa procurou desenvolver um material alternativo que seguisse padrões inovadores e sustentáveis. Assim, desenvolveu-se um composto cuja matriz é o amido e o reforço é o resíduo de papel.

O amido foi selecionado por ser oriundo de fonte renovável, ser biodegradável, estar disponível na sua forma natural e em grande quantidade, possuir baixo custo e aceitar incorporação de fibras vegetais ou resíduos sólidos industriais, possibilitando sua aplicação em produtos voltados a sustentabilidade. $\mathrm{O}$ amido pode ser extraído de diversas fontes, tais como, milho, mandioca, batata (CALEGARI et al., 2017 (a)).

Como carga do compósito, utilizou-se os resíduos de papéis oriundos do processo de impressão da indústria gráfica, para possibilitar outras formas de reciclagem a este material residual.

A inserção da carga em um compósito pode melhorar a sua performance, mas ainda existe restrições em relação ao desempenho do material durante sua vida útil. No caso de materiais compósitos reforçados com fibras vegetais, verifica-se que são capazes de absorver umidade em atmosfera úmida e/ou quando imerso em água, produzindo efeitos na interface fibra/matriz que leva a uma pobre eficiência da transferência de tensões. Além disso, a absorção de umidade pelas fibras naturais afeta suas propriedades físicas, mecânicas e térmicas (AZWA et al., 2013).

A celulose encontrada no papel é de natureza hidrofílica (SHANKAR \& RHIM, 2018). Considerando que o amido e o papel possuem essa natureza, pode ocorrer limitações na aplicação deste biocompósito, caso não seja criada uma barreira a fim de restringir a absorção de umidade. A submissão deste material a condições extremas de umidade pode acarretar mudanças nas propriedades do material, sendo a principal consequência o inchamento que afeta suas características (ELEOTÉRIO, 2000).

De acordo com Calegari et al. (2017 (b)), a absorção de umidade constitui-se como um fator limitante nos materiais hidrofílicos, no sentido de contornar este aspecto, podem ser utilizados revestimentos especiais. 
Segundo Shirmohammadli (2018) há um crescente interesse em usar substancias derivadas da natureza como substrato para impermeabilizantes, que podem reduzir os riscos ambientais. Na escolha dos impermeabilizantes utilizados neste estudo, houve a preocupação de buscar-se materiais não agressivos a natureza.

Os impermeabilizantes sintéticos, como a resina de base amina e de base fenólica, são usados para recobrir materiais hidrofílicos mas possuem inconvenientes relativos a emissão de formaldeído que é cancerígeno e ao mesmo tempo poluem o ambiente (THIENEN \& SPEE, 2008).

Assim, buscando definir um revestimento que torne o produto hidrofóbico, mantendo suas características sustentáveis, o objetivo deste trabalho foi testar a influência de diferentes impermeabilizantes na absorção de água no biocompósito amido/papel desenvolvido.

\section{Materiais e Métodos}

Inicialmente foi desenvolvido o biocompósito amido/papel e confeccionadas as amostras que, posteriormente, foram impermeabilizadas com quatro produtos comerciais diferentes. Logo após foram avaliadas quanto à absorção de água e da variação dimensional do material. Ainda, foram analisadas por microscopia eletrônica de varredura para verificar a morfologia do material antes e após a impermeabilização.

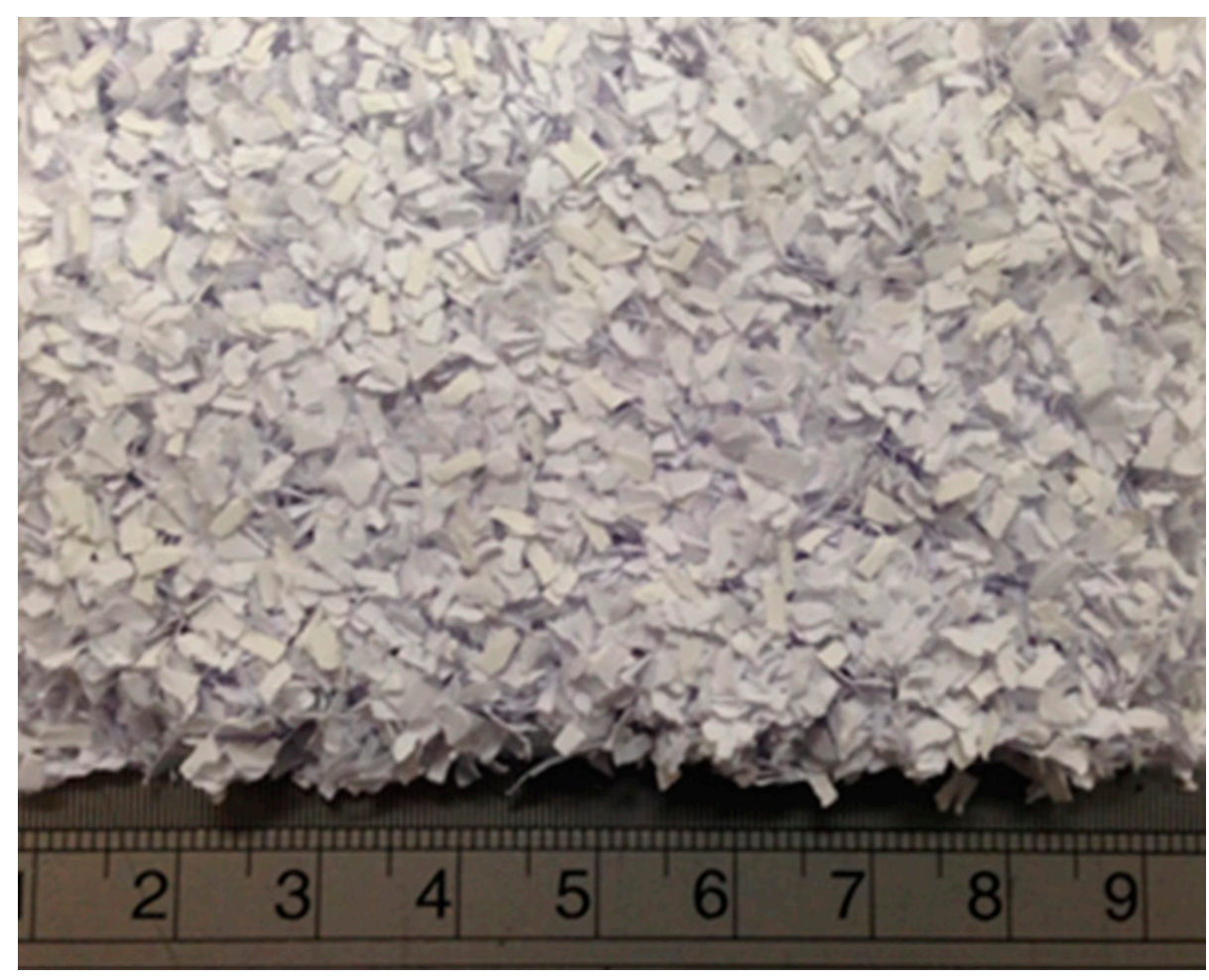

Fig 1. Imagem do resíduo de papel

Fonte: O autor, 2019

DATJournal v. 5 n. 12020 
Materiais

Para esta experiência utilizou-se o compósito amido/papel, constituído de água, amido de mandioca e resíduos de papéis provenientes do processo de impressão da Gráfica da UFRGS, dos resíduos gerados pela coladeira Eurobind, equipamento do setor de acabamento, da marca Heidelberg. Os resíduos de papel são apresentados na Figura 1. A matéria prima destas aparas é o papel offset da International Paper, cuja gramatura é de $75 \mathrm{~g} / \mathrm{m}^{2}$.

Na gelatinização do amido utilizou-se um recipiente com água quente, medida por um termômetro digital da marca BBQ e a estufa da marca DELEO 2A SED para a secagem do composto. Para manter uma espessura constante o material foi passado em uma laminadora de marca ARKE SF-300.

Para a impermeabilização do compósito amido/papel foram utilizados quatro tipos de impermeabilizantes:

- Resina AGT 1315 (fabricante Imperveg) - resina a base de poliuretano vegetal derivada de óleo de mamona, bicomponente, formulada pela mistura a frio de um prépolímero (componente A) e um poliol (componente B), utilizada, conforme fabricante, nas proporções de 1:1,5, indicada como matriz para o desenvolvimento de compósitos (IMPERVEG, 2019(a)).

- Resina UG 132A (fabricante Imperveg) - resina impermeabilizante a base de poliuretano vegetal (originado do óleo de mamona), bicomponente, formulada pela mistura a frio de um pré-polímero (componente A) e um poliol (componente B), utilizada nas proporções de 1:2, indicada para Impermeabilização interna de galerias e dutos de água (IMPERVEG, 2019(b)).

- Verniz ECOLAC (fabricante Salisil) - verniz a base de água, indicado para aplicação artesanal ou industrial em todos os tipos de madeira, MDF, compensados, cerâmica, vime, cortiça, gesso, isopor, couro, etc. Com alta transparência e solidez à luz. Não possui solventes na sua composição. Exala baixíssimo odor e minimiza os riscos à saúde do aplicador. Ocasiona baixa emissão de compostos orgânicos voláteis (VOC), não agride ao meio ambiente (NOVA SANTA MARIA TINTAS, 2018).

- Verniz Marítimo (Fabricante Montana) - verniz transparente, de acabamento brilhante ou acetinado, desenvolvido de resina à base de poliuretano e filtro solar, que atribui ao verniz resistência aos raios ultravioleta, resistência ao atrito e intemperes. Indicado para aplicação em madeira. 


\section{Métodos}

Para o estudo de impermeabilização, desenvolveu-se o compósito amido/papel e posteriormente foram confeccionados os corpos de prova que foram submetidos a recobrimentos de diferentes naturezas. Estes foram submersos em água para a análise do comportamento dos impermeabilizantes. Devido à ausência de normas padronizadas para este material, os níveis de absorção de água foram avaliados conforme os parâmetros de classificação das placas cerâmicas (NBR 13.818 (Associação Brasileira de Normas Técnicas, 1997)): baixa absorção (0 a 3\%), média absorção (3 a 6\%) e alta absorção ( $\geq 6 \%$ ) (VOITILLE, 2018).

\section{Desenvolvimento do compósito amido/papel}

O desenvolvimento do compósito amido/papel iniciou com a gelatinização do amido (para a ruptura da sua estrutura cristalina), por meio do seu aquecimento em um recipiente com água em uma temperatura de $\approx 90^{\circ} \mathrm{C}$, que possibilitou transformá-lo em um material no estado de gel. $\mathrm{O}$ amido gelatinizado foi incorporado ao papel e ao amido seco manualmente (Figura 2 a), de forma rápida e constante. A proporção em massa entre os constituintes foi de $27 \%$ de amido gelatinizado, $37 \%$ de amido seco e $36 \%$ de papel seco. Após a mistura, o material foi laminado para garantir uma espessura constante. (Figura 2 b) (CARDOSO et al., 2017).

Fig 2. (a) Preparação do material (amido gelatinizado, amido seco e papel), (b) Compósito amido/papel. Fonte: $\mathrm{O}$ autor, 2019
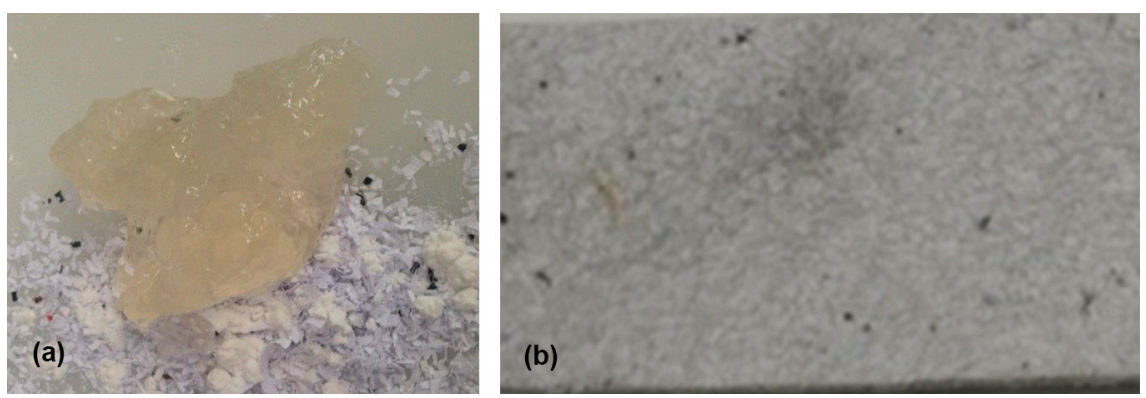

Em seguida, foram confeccionados os corpos de prova, conforme as normas exigidas para cada ensaio. Todos estes procedimentos foram executados no Laboratório de Desenvolvimento Integrado de Materiais e Produtos (DIMP) do Instituto Federal Sul-rio-grandense (IFSul/ Sapucaia do Sul). 


\section{Confecção dos corpos de prova}

Após a laminação, processo utilizado para garantir uma espessura constante e melhor uniformidade, colocou-se o material em uma superfície plana, e com o auxílio de um paquímetro digital (marca Digimess resolução $0.02 \mathrm{~mm}$ ) e um estilete cortou-se os corpos de prova, que foram levados à estufa da marca DELEO 2A SED para a secagem do compósito, a $90{ }^{\circ} \mathrm{C} / 1$ hora para evaporação da água.

As amostras foram confeccionadas em um total de 39 amostras, sendo utilizado 3 amostras para cada família, conforme a Tabela 1, foram divididas em 5 grupos, denominados amostra sem impermeabilizante (AP), amostra com resina AGT 1315 (A), amostra com resina UG 132A (U), amostra com verniz Ecolac (E) e amostra com verniz marítimo (V). Na primeira coluna da Tabela 1 apresenta-se a identificação dos corpos de prova, as letras maiúsculas referem-se ao tipo de material, o número representa a quantidade de demãos e a letra minúscula é a identificação do corpo de prova dentro da família. Conforme a Tabela 1, para revestir os corpos de prova com os impermeabilizantes utilizou-se um pincel. Antes e após cada demão as amostras foram pesadas e medidas.

\begin{tabular}{lll}
\hline $\begin{array}{l}\text { Identificação dos corpos } \\
\text { de prova }\end{array}$ & Recobrimento (demão) & Impermeabilizantes \\
\hline AP0a & Sem recobrimento & - \\
AP0b & & \\
AP0c & 1 demão & Resina poliuretana à base de óleo \\
\hline A1a, A1b, A1c; & 2 demão & de mamona AGT1315 \\
A2a, A2b, A2c; & 3 demão & Resina poliuretana à base de óleo \\
A3a, A3b, A3c; & 1 demão & de mamona UG 132A \\
\hline U1a, U1b, U1c; & 2 demão & \\
U2a, U2b, U2c; & 3 demão & Ecolac \\
U3a, U3b, U3c; & 1 demão & \\
\hline E1a, E1b, E1c; & 2 demão & Verniz marítimo (base de \\
E2a, E2b, E2c; & 3 demão & poliuretano) \\
E3a, E3b, E3c; & 1 demão & \\
\hline V1a, V1b, V1c; & 2 demão & 3 demão \\
V2a, V2b, V2c; & & \\
V3a, V3b, V3c; & &
\end{tabular}

Tabela 1. Descrição dos corpos de prova com os respectivos impermeabilizantes.

Fonte: $\mathrm{O}$ autor, 2019

\section{Ensaio de absorção de água}

Para este ensaio de absorção de água foi utilizada a Norma EN 317 (Normalización Espanõla, 1993) que se refere à determinação do inchamento em espessura após imersão em água. Conforme esta norma, os corpos de prova devem possuir dimensões de aproximadamente $50 \mathrm{~mm}$ x $50 \mathrm{~mm}$. As amostras foram pesadas em balança analítica (marca Pantec modelo FA2104N), medidas as espessuras em um micrômetro (marca Digimess reso- 
lução 0,001 mm). Após este procedimento os corpos de prova foram preparados para o ensaio. Os corpos de prova identificados na coluna 1 da Tabela 1 foram imersos em água à temperatura ambiente. Após 2 horas de imersão as amostras foram retiradas do recipiente e removidas o excesso de água com auxílio de papel toalha, logo após foi realizada novamente a pesagem. Em seguida foram recolocadas no recipiente com água por mais 22 horas para completar às 24 horas de imersão e realizado o mesmo procedimento anterior finalizando o ensaio.

Os valores em massa dos corpos de prova foram registrados para determinação da absorção de água (Equação 1).

$$
\mathrm{A} \%=100 \times\left(\mathrm{M}_{2}-\mathrm{M}_{1}\right) / \mathrm{M}_{1}
$$

Onde, $\mathrm{A} \%$ é a absorção de água em percentual, $\mathrm{M}_{1}$ é a massa inicial da amostra em gramas, antes da inserção na água, e $M_{2}$ é a massa da amostra em gramas, após a inserção em água.

Neste estudo, a Equação (1) foi utilizada para 2 e 24 horas de imersão em água a temperatura ambiente.

\section{Análise Estatística}

Após a realização do ensaio de absorção de água os resultados foram analisados com a ANOVA fator único considerado um nível de confiança de $95 \%$, sendo assim, se o valor de "p" for menor que 0,05 as famílias são diferentes (TARRÍO-SAAVEDRA et al., 2011). O resultado da análise da variância está representado nos gráficos de absorção de água através de letras.

\section{Resultados}

Nos testes de impermeabilização realizados com o compósito Amido/papel buscou-se analisar o comportamento do material em relação à absorção de água com quatro diferentes impermeabilizantes tendo-se como referência o comportamento das amostras sem o produto. A escolha destes impermeabilizantes deve-se às características fornecidas pelos fabricantes, na qual fatores como, inexistência de metais pesados, substâncias não tóxicas e solubilidade em água, foram decisivos para a escolha, porque se aproximam dos aspectos sustentáveis do compósito desenvolvido. A composição dos impermeabilizantes dos vernizes $\mathrm{E} e \mathrm{~V}$ são à base de água e os $\mathrm{A}$ e U são resinas de poliuretano vegetal a base de óleo de mamona. 
A Tabela 2 apresenta os valores médios de absorção de água em $2 \mathrm{~h}$ e $24 \mathrm{~h}$ encontrados para as famílias de amostras produzidas neste artigo. A seguir são apresentados os resultados individuais para cada tipo de impermeabilizante. Conforme será visto nas próximas figuras, amostras com um percentual de absorção de água inferior a $0,1 \%$ estão representadas por asterisco na legenda.

\begin{tabular}{lllllll}
\hline \multicolumn{5}{c}{ 2 Horas } & \multicolumn{3}{l}{ 24 Horas } \\
\hline AP & \multicolumn{3}{c}{ 0 Demão } & \multicolumn{3}{l}{ 0 Demão } \\
\hline & 1 Demão & 2 Demão & 3 Demão & 1 Demão & 2 Demão & 3 Demão \\
\hline A & $2,84 \pm 0,85$ & $0,51 \pm 0,81$ & $0,00 \pm 0,00$ & $28,90 \pm 4,90$ & $4,74 \pm 3,72$ & $0,04 \pm 0,05$ \\
\hline U & $2,38 \pm 0,10$ & $0,00 \pm 0,00$ & $0,00 \pm 0,00$ & $24,80 \pm 3,56$ & $1,40 \pm 0,13$ & $0,04 \pm 0,00$ \\
\hline V & $1,18 \pm 0,24$ & $0,61 \pm 0,08$ & $0,00 \pm 0,00$ & $27,49 \pm 4,14$ & $4,32 \pm 0,02$ & $2,20 \pm 0,17$ \\
\hline E & $11,63 \pm 1,79$ & $1,35 \pm 0,20$ & $0,11 \pm 0,11$ & $60,54 \pm 11,51$ & $8,19 \pm 0,44$ & $5,23 \pm 0,12$ \\
\hline
\end{tabular}

Tabela 2. Valores médios em percentual de absorção de água dos materiais desenvolvidos. Fonte: O autor, 2019

Ensaio de absorção de água nas amostras sem revestimento (AP) no compósito Amido/papel

Primeiramente, foram submetidas ao ensaio de absorção de água as amostras que não passaram pelo processo de impermeabilização. Pela análise dos dados, pode-se observar que nas amostras sem recobrimento AP0a, AP0b, AP0c, após 2 horas inseridos na água, houve um aumento percentual médio de $60,68 \%$ em massa das amostras. 0 material perdeu sua consistência e houve dificuldade para verificar a massa das amostras que foram retiradas da água após 2 horas de imersão. Em função deste comportamento, as amostras sem impermeabilização não foram submetidas aos experimentos de 24 horas de imersão. Esses resultados evidenciam o caráter hidrofílico deste material.

\section{Revestimento com verniz Ecolac (E) no compósito Amido/papel}

Este impermeabilizante, para todas as condições apresentadas na Tabela 1, foi o que demonstrou os piores resultados. Após $2 \mathrm{~h}$ submersas em água, as amostras já apresentavam pegajosidade e proporcionaram uma sensação de estarem úmidas. Em $24 \mathrm{~h}$, com uma demão, as amostras ficaram totalmente deformadas e um índice de absorção de 60,54 \% de água foi constatado. Assim, houve um aumento considerável na absorção de água após 


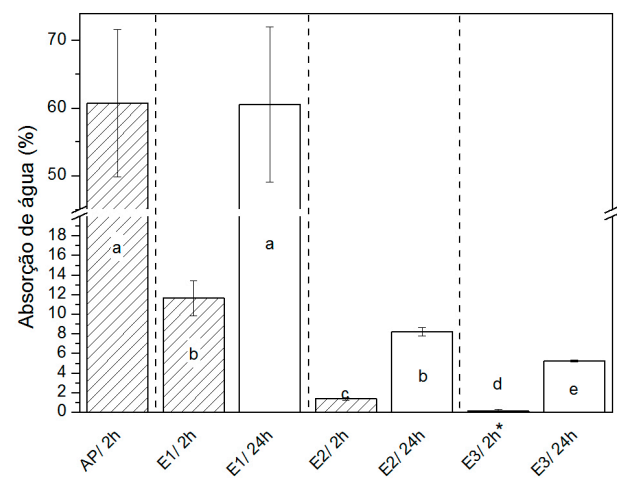

Fig 3. Efeito do impermeabilizante E na absorção de água do compósito. Fonte: O autor, 2019

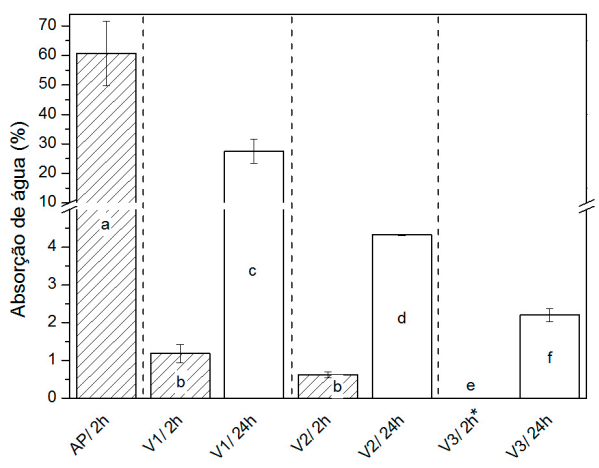

Fig 4. Efeito do impermeabilizante $\mathrm{V}$ na absorção de água do compósito. Fonte: $\mathrm{O}$ autor, 2019

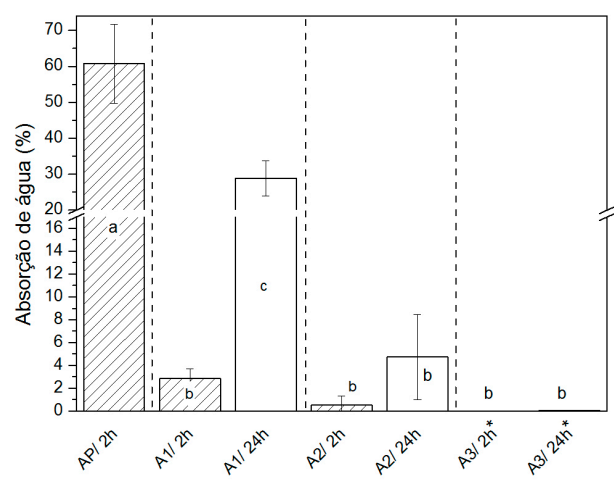

Fig 5. Efeito do impermeabilizante A na absorção de água do compósito. Fonte: $\mathrm{O}$ autor, 2019
$24 \mathrm{~h}$, o que evidencia a ineficiência deste impermeabilizante para reduzir a habilidade de absorção de água apresentada originalmente pelo compósito Amido/papel. Pelos resultados apresentados na ANOVA constata-se que com uma demão, em $24 \mathrm{~h}$, o material absorveu uma quantidade equivalente ao material sem recobrimento por $2 \mathrm{~h}$.

Conforme a Figura 3, em 24 horas, para duas e três demãos as amostras apresentaram uma redução na absorção de água em relação a uma demão e, apesar das amostras se manterem visualmente iguais ao estado inicial, absorveram até $68 \%, 8 \%$ e $5 \%$ de água, para uma demão, duas demãos e três demãos respectivamente.

\section{Revestimento com verniz marítimo (V) no compósito Amido/papel}

O revestimento com verniz marítimo alcançou níveis satisfatórios, de acordo com a norma NBR 13.818 (Associação Brasileira de Normas Técnicas, 1997), para duas e três demãos em $2 \mathrm{~h}$, mas não correspondeu nos resultados obtidos em $24 \mathrm{~h}$, para uma e duas demãos, só alcançando um índice inferior a 3\% com três demãos. Isto percebe-se na Figura 4 pela ANOVA. Quanto a aparência das amostras verificou-se que para uma demão ao término de $24 \mathrm{~h}$ houve um inchamento em algumas regiões próximas aos vértices das amostras. Já para duas e três demãos as amostras não apresentaram nenhuma alteração visível.

Revestimento com PU da mamona AGT 1315 (A) no compósito Amido/papel

Neste conjunto de amostras observou-se que com apenas uma demão houve uma média de absorção da água de $2,84 \%$ quando os corpos de prova ficaram inseridos na água pelo período de $2 \mathrm{~h}$. Nestas condições, visualmente, não foram verificadas alterações aparentes na superfície das amostras provenientes da absorção de água, além disso, elas se mantiveram iguais e não ficaram pegajosas. Já, quando foram inseridas por $24 \mathrm{~h}$, houve uma absorção de água média de $28,90 \%$, e as amostras apresentaram-se deterioradas nos vértices.

Nas amostras com duas demãos que foram submetidas a $2 \mathrm{~h}$ de exposição a água, absorveram um valor médio $0,51 \%$ de água e na sua aparência não foram observadas deformidades em função dessa absorção. No período de $24 \mathrm{~h}$ também não houve mudanças aparentes, isto é, as amostras se mantiveram iguais apesar do aumento de absorção de água que foi de $4,74 \%$, ocorrendo o mesmo comportamento nas amostras com três demãos, em que foram encontrados valores médios de absorção de 0,00 e $0,04 \%$ nos períodos de $2 \mathrm{~h}$ e $24 \mathrm{~h}$. 
A Figura 5 apresenta os valores médios de absorção de água comparando a amostra sem recobrimento com as amostras impermeabilizadas com o revestimento PU da mamona AGT 1315, nos períodos de $2 \mathrm{~h}$ e $24 \mathrm{~h}$. Pela análise da Figura 5, verifica-se que, a eficiência do impermeabilizante ocorreu com as amostras de duas demãos, apresentando resultado estatisticamente igual a partir de duas horas de imersão.

\section{Revestimento com a resina UG 132 A (U), no compósito Amido/papel}

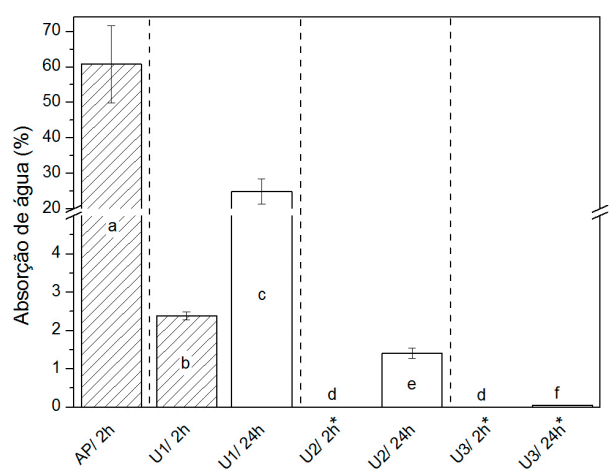

Fig 6. Efeito do impermeabilizante U na absorção de água do compósito. Fonte: $\mathrm{O}$ autor, 2019
Neste procedimento observou-se que nas amostras com uma demão de $2 \mathrm{~h}$ e $24 \mathrm{~h}$ os índices foram semelhantes ao impermeabilizante A, obtiveram um índice acima de $2 \%$ para $2 \mathrm{~h}$ e acima de $24 \%$ em $24 \mathrm{~h}$, não obtendo uma impermeabilização satisfatória em $24 \mathrm{~h}$. Nestas condições, visualmente, foram verificadas alterações aparentes na superfície das amostras, bem como pegajosidade após a absorção de água.

Nas amostras de duas e três demãos, para $2 \mathrm{~h}$ e $24 \mathrm{~h}$, este produto apresentou características de baixa absorção de água de acordo com a metodologia utilizada neste trabalho. Sendo assim, pode-se afirmar que para duas demão, este material tem uma excelente eficiência sendo utilizado em contato com a umidade.

A Figura 6 apresenta os valores médios de absorção de água comparando a amostra sem recobrimento com as amostras impermeabilizadas com o revestimento PU da mamona UG 132 A (U) de acordo com as especificações da Tabela 1, nos períodos de $2 \mathrm{~h}$ e $24 \mathrm{~h}$. Pela análise da Figura 6, no período estudado, verifica-se, a partir da análise da ANOVA, que a eficiência do impermeabilizante também ocorre a partir de duas demãos, tanto para $2 \mathrm{~h}$ quanto para $24 \mathrm{~h}$, conforme a norma NBR 13.818 (Associação Brasileira de Normas Técnicas, 1997).

Ao comparar as Figuras 5 e 6, verifica-se um menor desvio padrão na Figura 6 que pode estar relacionado à menor viscosidade do impermeabilizante que facilitou a melhor cobertura da amostra.

\section{Análise comparativa entre os impermeabilizantes utilizados}

A grande absorção de água observada nos materiais biodegradáveis produzidos (na ordem de 60\%) e a perda de integridade do mesmo com esse nível de absorção evidenciam a necessidade de realizar procedimentos de impermeabilização. Caso contrário, teriam um número muito restrito de produtos nos quais poderiam ser aplicados.

Os resultados obtidos neste estudo permitiram definir o impermeabilizante e o número de demãos necessárias para obter-se uma proteção eficiente em relação a absorção de água. Além disso, como apresentado na literatura, além de minimizar a absorção de água, o uso de impermeabilizantes pode evitar a ocorrência de micro-organismos no material criado, 
que, em função da ação combinada da água e da temperatura, podem reduzir a vida útil do material durante o seu uso (KOENIG \&HUANG, 1994).

Quanto aos impermeabilizantes estudados, pode-se afirmar que os resultados evidenciam que o uso de três demãos foram suficientes para alcançar uma cobertura eficiente para resistir a imersão em água por $2 \mathrm{~h}$, conforme demonstrado na Tabela 2.

Os índices de absorção de água com uma demão, no período de $24 \mathrm{~h}$, foram altos para todas as amostras, demonstrando que, dependendo dos requisitos de resistência à água necessários para aplicação deste material, será inevitável a realização de duas ou três demão de impermeabilizante para obter-se uma cobertura mais eficiente.

Comparando os resultados dos impermeabilizantes A, U, E com o V para 2 horas em uma demão, observa-se que este produto obteve os melhores resultados em relação aos demais, mas ressalta-se que os resultados de A e U ainda estão dentro dos padrões de baixa absorção (0 a 3\%).

Os impermeabilizantes $\mathrm{V}$ e $\mathrm{E}$ apesar de absorverem mais água que os A e U demonstraram para duas e três demãos índices de absorção bem menores que para uma demão em $24 \mathrm{~h}$, isto é, uma redução de 15,6\% e 13,5\% para duas demãos e $8 \%$ e 8,6\% para três demão respectivamente em relação a uma demão, mas ainda não chegando a índices aceitáveis de impermeabilização para o impermeabilizante $\mathrm{E}$ conforme a metodologia apresentada neste trabalho.

Já, com três demãos, entre os impermeabilizantes testados, conforme os resultados apresentados na Figura 7 os revestimentos A e U foram os que demonstraram o melhor desempenho conforme a ANOVA.

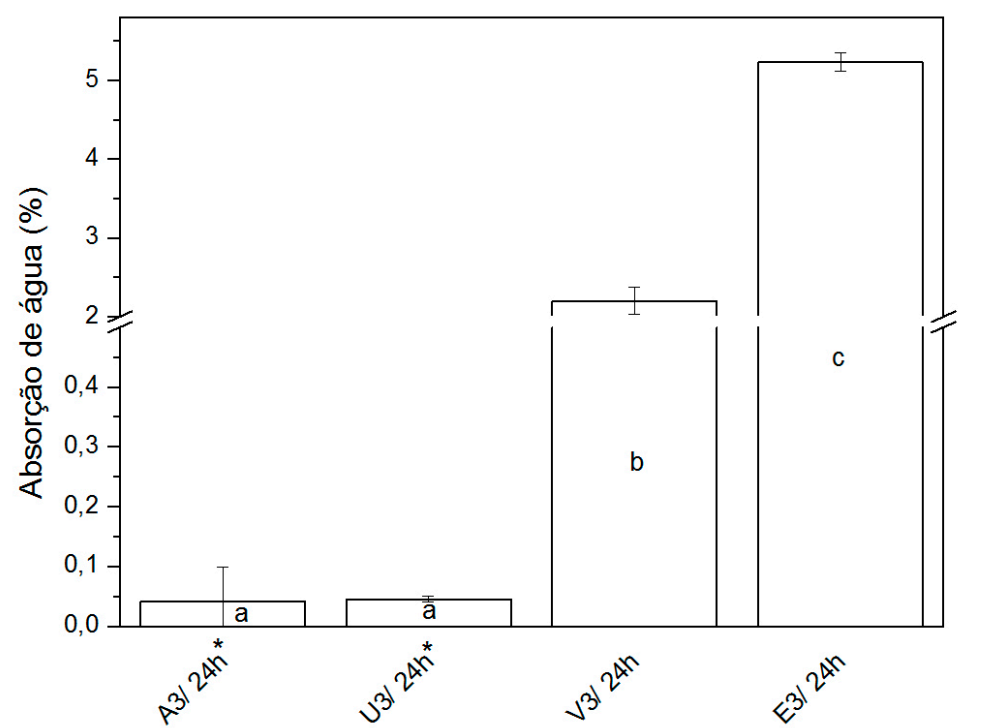

Fig 7. Comparação da absorção de água dos impermeabilizantes para 3 demãos/24 horas. Fonte: O autor, 2019
Finalmente, na análise entre todos os impermeabilizantes com duas demãos em $24 \mathrm{~h}$, observa-se que o impermeabilizante $U$ de acordo com a norma NBR 13.818 (Associação Brasileira de Normas Técnicas, 1997) apresenta baixa absorção, obtendo o melhor resultado. Além disso, para uma demão, 2 horas, foi o que apresentou o mais baixo padrões de absorção, podendo-se afirmar que este impermeabilizante obteve os melhores resultados quanto ao número de demãos que são necessárias para impermeabilizar este material e, conforme o fabricante é um material inerte e não tóxico, que pode ser utilizado em contato com água potável e alimentos (IMPERVEG, 2019 (c)). 


\section{Considerações Finais}

A criação de novos materiais biodegradáveis é uma aposta para a inserção de materiais e produtos mais sustentáveis. 0 estudo da impermeabilização deste compósito amido/papel buscou promover novas opções de materiais que contribuam com os aspectos ambientais e viabilizar a ampliação do uso deste material de caráter hidrofílico, buscando-se a redução da absorção de água.

Neste sentido, foram analisados quatro impermeabilizantes sob as amostras com uma, duas e três demãos, em $2 \mathrm{~h}$ e $24 \mathrm{~h}$ de imersão em água, com o objetivo de verificar a eficácia dos recobrimentos, a viabilidade de proteger-se um material hidrofílico com substratos menos agressivos ao meio ambiente.

Pelo estudo, os impermeabilizantes que melhor corresponderam a estes objetivos foram o A e o U, que obtiveram os melhores índices entre os impermeabilizantes analisados. Os outros impermeabilizantes também obtiveram bons resultados com duas e três demãos, mas não são indicados para um período prolongado de exposição à umidade.

Pelo exposto, pode-se concluir que o material criado, o compósito amido/papel, pode ser revestido com estes impermeabilizantes, que obterá características hidrofóbicas e poderá ter aplicação na produção de artefatos que estejam expostos ao meio ambiente.

Entretanto, o impermeabilizante U, além de obter-se maior economia em relação ao número de demãos que são suficientes para obter-se a sua eficácia, este produto, por ser a base de poliuretano do óleo de mamona, portanto, de fonte renovável, poderá ser indicado o seu uso neste novo material, pois manteria as características sustentáveis deste compósito desenvolvido.

\section{Agradecimentos}

Os autores agradecem a empresa Imperveg Polímeros Indústria e Comércio Ltda. pela doação das resinas Imperveg AGT 1315 e Imperveg UG 132A. 


\section{Referências}

ASSOCIAÇÃO BRASILEIRA DE NORMAS TÉCNICAS. NBR 13.818: Placas Cerâmicas para Revestimento - Especificação e Métodos de Ensaio (descrição dos parâmetros dos ensaios), Rio de Janeiro, maio 1997.

AZWA, Z.N.; YOUSIF, B.F.; MANALO, A.C.; KARUNASENA, W. A review on the degradability of polymeric composites based on natural fibres. Materials \& Design, v.47, p.424-442, 2013. CALEGARI, E.P. et al. Composites from renewable resources with thermoplastic starch matrix. In: Asim Shahzad. (Org.). Biocomposites: Properties, Performance and Applications. 1 ed. New York: Nova Science Publishers, 2017, p. 79-92 (a).

CALEGARI, E.P.; PORTO, J.S.; NEJELISKI, D.M.; DUARTE, L.C.; OLIVEIRA, B.F. Experimental study on waterproofing MDF with castor oil-based vegetal polyurethane. Matéria, v.22, p. 1-10, 2017 (b).

CARDOSO, G.; ANGRIZANI, C.C.; PORTO, J.S.; CALCAGNO, C.I.W. Reutilização de resíduos de papéis em composição com o amido: uma opção de material para o design sustentável. In: $6^{\circ}$ Seminário de Inovação e Tecnologia do IFSul (Inovtec), Sapucaia do Sul, 2017.

ELEOTÉRIO, J. R. Propriedades físicas de painéis MDF de diferentes densidades e teores de resina. 2000. 121f. Dissertação de mestrado - Escola Superior de Agricultura "Luís de Queiroz", Universidade de São Paulo, Piracicaba, São Paulo, 2000.

FARUK, O.; BLEDZKI, A.K.; FINK, H.P.; SAIN, M. Biocomposites reinforced with natural fibers: 2000-2010. Progress in Polymer Science, v.37, nº11, p.1552-1596, 2012.

IMPERVEG. Ficha Técnica IMPERVEG AGT 1315. (http://imperveg.com.br/wp-content/ uploads/2015/05/FICHA-T\%C3\%89CNICA IMPERVEG-AGT-1315.pdf) (a).

IMPERVEG. Ficha Técnica IMPERVEG UG 132A. (http://imperveg.com.br/wp-content/ uploads/2015/04/FICHA_TECNICA_IMPERVEG_UG132_A1.pdf) (b).

IMPERVEG. Poliuretano Vegetal Imperveg. (http://imperveg.com.br/poliuretano-vegetal-imperveg/) (c).

KOENIG, M.F.; HUANG, S.J. Evaluation of crosslinked poly(caprolactone) as a biodegradable, hydrophobic coating. Polymer Degradation and Stability, v.45, no1, p.139-144, 1994. NORMALIZACIÓN ESPAÑOLA. Norma EN 317: Tableros de partículas y tableros de fibras. Determinación de la hinchazón en espesor después de inmersión en agua. (Versión oficial EN 317:1993).

NOVA SANTA MARIA TINTAS. Verniz Ecolac. (http://www.novasantamaria.com.br/ecolac-verniz-a-base-dagua).

PICCOLI, M.; PACHECO, J.; CÂNDIDO, L.H.A.; BRANDI, L.B. Reutilização de tubos de papelão: estudo da impermeabilização visando sua aplicação no design de produtos. In: $11^{\circ}$ Congresso Brasileiro de Pesquisa e Desenvolvimento em Design, Gramado, 2014.

SANJAY, M.R.; ARPITHA, G.R.; YOGESHA, B. Study on mechanical properties of natural - glass fibre reinforced polymer hybrid composites: a review. Materials Today: Proceedings, v.2, p. $2959-2967,2015$.

SHANKAR, S.; RHIM, J-W. Effects of poly(butylene adipate-co-terephthalate) coating on the water resistant, mechanical, and antibacterial properties of Kraft paper. Progress in Organic Coatings, v.123, p.153-159, 2018.

SHIRMOHAMMADLI, Y.; EFHAMISISI, D.; PIZZI A. Tannins as a sustainable raw material for green chemistry: A review. Industrial Crops and Products, v.126, p.316-332, 2018. 
TARRÍO-SAAVEDRA, J.; NAYA, S.; FRANCISCO-FERNÁNDEZ, M.; ARTIAGA, R.; LOPEZ-BECEIRO, J. Application of functional ANOVA to the study of thermal stability of micro-nano silica epoxy composites. Chemometrics and Intelligent Laboratory Systems, v.105, nำ1, p.114-124, 2011.

THIENEN, G.V.; SPEE, T. Health Effects of construction materials and construction products. Tijdschrift voor toegepaste Arbowetenschap, nำ, p.2-23, 2008.

VOITILLE, N. Impermeabilidade: Graus de Absorção de Água, 2018. (http://www.cliquearquitetura.com.br/artigo/ceramicas-impermeabilidade--resistencia.html). 